\title{
Optimizing Lighting in an Office for Demand Response Participation Considering User Preferences
}

\author{
Mahsa Khorram, Pedro Faria, Zita Vale \\ Polytechnic of Porto (IPP), Porto, Portugal \\ makgh@isep.ipp.pt, pnf@isep.ipp.pt, zav@isep.ipp.pt
}

\begin{abstract}
The world is moving toward demand response programs, energy optimization, and using renewable energy resources more than the past. Buildings are responsible for a significant part of energy consumption and they are considered as suitable cases for reducing energy usage. Therefore, energy management in buildings should be improved. This paper presents a multi-period optimization algorithm with the aim of reducing power consumption of the lights and maintain user comfort. For this purpose, several comfort constraints are considered, and several parameters are defined. The case study of the paper uses real data monitored by an implemented automation infrastructure in a building. Four scenarios are presented to validate the impacts of proposed algorithm. Each scenario surveys different aspects of user comfort constraint and the obtained results illustrate the performance of algorithm.
\end{abstract}

Index Terms - Energy Optimization, Office Building, Lighting System, User Comfort.

\section{NOMENCLATURE}

Parameters
Priority
$P$
RR
PRR
Init.P
MaxRed_R
MaxRed_T
Indexes
L
$T$
$R$

Importance weight of Light

Power of Light

Required power Reduction

Power Reduction Rate

Initial power of Light

Maximum power reduction in each room

Maximum power reduction in each period

\begin{abstract}
Maximum number of Lights
Maximum number of Periods

Maximum number of Rooms
\end{abstract}

\section{INTRODUCTION}

\section{A. Motivation and Background}

Energy consumption has always been a concern for worlds during various times [1]. The increment of energy consumption in the last decades has made irreparable consequences. Environmental problems such as global warming, melting glaciers, seasons replacements in many countries are clear examples of the aftermath of fossil fuels usage [2]. Many efforts have been done and many solutions have been proposed by the experts in this field, but each proposed solution have foibles

The present work was done and funded in the scope of the following projects COLORS Project PTDC/EEI-EEE/28967/2017 and UID/EEA/00760/2019 funded by FEDER Funds through COMPETE program and by National Funds through FCT. which still make the energy consumption topics challenging. Renewable Energy Resources (RER) are presented as clean energy and can be considered as an alternative for fossil fuels however their uncertainty and stochasticity require comprehensive and accurate planning [3]. In this context, Demand Response (DR) programs organize the user's consumption pattern according to electricity price variations or technical issues with considering consumers and producers interests [4]. DR programs have a desirable variety which is divided into two main groups, namely price-based DR and incentive-based DR [5]. All types of buildings including domestic, industrial, commercial, and office buildings are responsible for $40 \%$ of world energy consumption [6], [7]. Office buildings can be considered as flexible options for implementing DR programs since usually, they have significant energy consumption, and in some cases can be more equipped to automation infrastructure than residential houses [8].

In order to implement the DR programs in office buildings, the lighting systems play an important role as dynamic and flexible loads since their consumption can be reduced or interrupted [9]. In this context, Supervisory Control And Data Acquisition (SCADA) system play a key role in DR implementation since it offers various advantages in order to have automatic load control in different types of buildings [10]. For instance, the SCADA system can dominate the illumination of the lighting system which they are fully controllable via the Digital Addressable Lighting Interface (DALI) [11].

DR programs and other operations that modify the user consumption pattern, directly effect on user comfort. Nowadays, one of the main challenges of energy consumption optimizers is maintaining the balance between goals of program and convenience of users. The three basic factors which determine the occupant's quality of lives in a building environment are thermal comfort, visual comfort and air quality [12]. The illumination level is used to indicate the visual comfort in a building environment.

\section{B. Relevant Literature}

Several studies have been done in the scope of consumption optimization in the buildings such as [11] that focuses on reducing power consumption of lights in an office building based on the importance of each light without considering user comfort constraints. The power consumption of lights and air conditioners have been optimized in order to reduce the final 
energy cost of the user in [13] under real-time pricing while the user convenience is not mentioned. In [14], the authors have been used the lights as the second priority for the algorithm beside air conditioners for reducing the power reduction. The authors in [15], have been proposed a particle swarm optimization algorithm that is controlling the lighting system of the large-scale buildings by using adjustable lights and wireless sensor networks, however, the user satisfaction is out of the scope of the article. The paper [16], proposes an intelligent and smart building that the optimum visual user preferences is predicted, and it claims that could be able to save a significant amount of energy compared to a normal situation.

\section{Contribution and Organization}

The present article proposes a multi-period optimization algorithm for minimizing the power consumption of the lighting system in an office building. The methodology is modeled as a linear problem by Optimization Modeling Package in Rstudio (OMPR). The algorithm attempts to maintain user comfort by considering several constraints and defining effective parameters. Each constraint applies restriction on various aspects that effect on user comfort.

After this introductory section, the optimization algorithm and the implemented methodology is explained in Section II. The case study with four scenarios are demonstrated in Section III in order to validate the performance of the proposed optimization algorithm, and the obtained results will be compared in section IV. Finally, Section VI describes the main conclusions of the work.

\section{MODEL DESCRIPTION}

This section describes the optimization algorithm which is implemented in the present SCADA system. The main purpose of the algorithm is minimizing the power consumption of the lights, while the user comfort is respected. It is important to achieve the desired power reduction of the algorithm, whenever the referred power reduction is reduced a balanced level from all the lights. For this purpose, several constraints and parameters are defined. One specific number between 0 and 1 is assigned to each light to show the priority of each light for the system. The definition of these priority numbers depends on several aspects such as preferences of the user, the location of each light, and the level of natural light. Fig. 1 shows the steps of the present algorithm with some details.

As it is presented in Fig. 1, the optimization algorithm starts with receiving input data such as power consumption of the lights, the nominal power consumption of the lights and total consumption of the building. The components of the SCADA system are obligated to monitor the initial data as well as controlling the lighting system. The priority numbers are decimal numbers between 0 and 1 determinant parameters that specify the importance of each light for the system. The priority numbers close to 1 are the most important lights for the system while the 0 means the last priority. Power Reduction Rate (PRR) is another determinative parameter for the algorithm. Since the present algorithm is a multi-periods optimization, PRR is defined to limit the power reduction of each specific light in all periods. It means that the total power reduction of each light during all periods is limited to PRR.

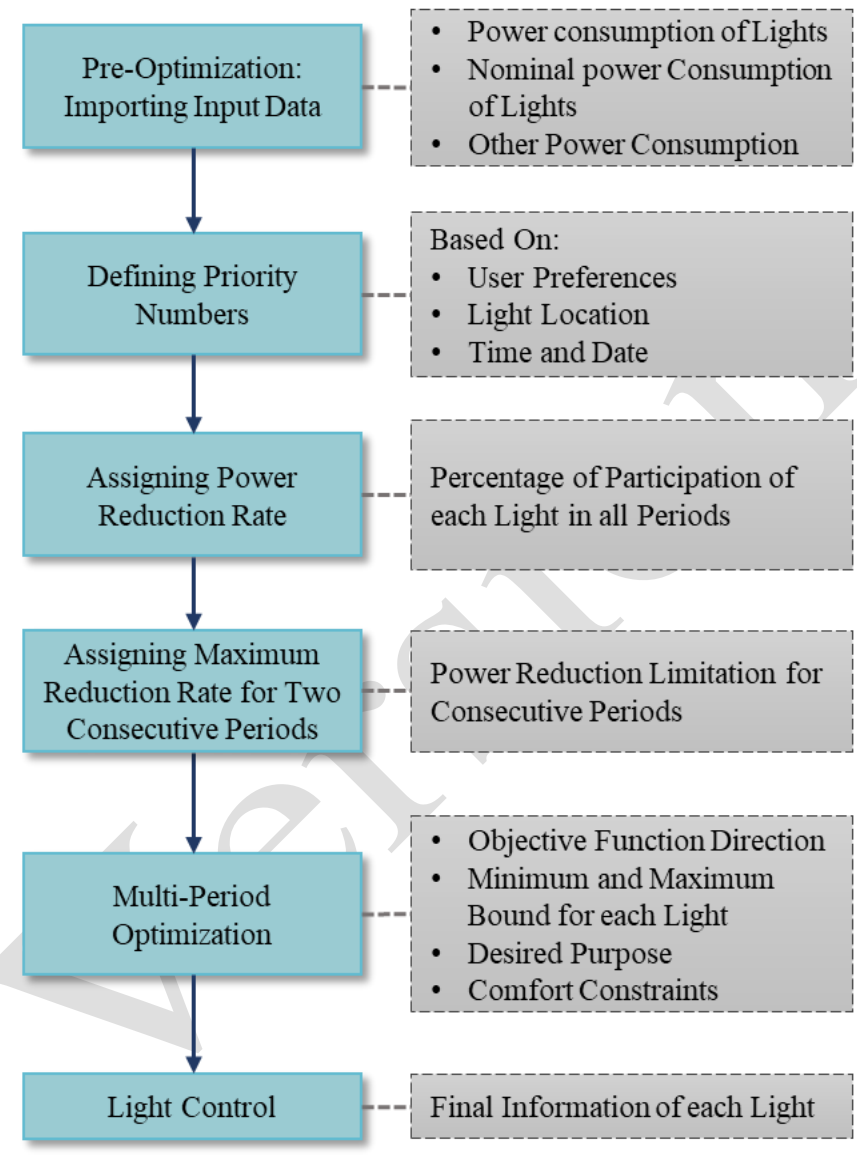

Fig. 1. The flowchart of the proposed optimization methodology.

Another parameter so-called Maximum Reduction Rate (MRR) is defined to avoid reducing power consumption from lights in consecutive periods. After specifying the parameters, the relative constraints should define, and the variables should bound. The desired purpose of the algorithm is minimizing a certain amount of power consumption from the lights. Defining the range of power reduction can be affected by several issues such as electricity price, ON-Peak or Off-Peak hours, the rate of power generation, and stored energy in batteries if exist. After defining all the required data, the algorithm runs, and the results will obtain.

The proposed methodology is defined as a Linear Programming (LP) optimization problem, which is modeled via "OMPR" package of Rstudio ${ }^{\circledR}$ (www.rstudio.com) and is solved via "GLPK" library.

The Objective Function (OF) of the proposed optimization algorithm is shown in (1) for minimizing the power consumption of the lights.

$$
\text { Minimize } O F=\sum_{t=1}^{T} \sum_{l=1}^{L} \text { Priority }_{(l, t)} \times P_{(l, t)}
$$

It should be mentioned that Priority is converted to decimal numbers between 0 and 1 . The small numbers indicate the nonpriority lights and the important lights have bigger. 
The defined range for Priority and $P$ is referred in the author's previous work [14] and they are not mentioned in this section. Equation (2) shows the desired power reduction of the system in each period that can be adjusted based on several aspects such as electricity price, power generations, and user prefrences.

$$
\begin{aligned}
& \sum_{l=1}^{L} P_{(l, t)}=R R_{(t)} \\
& \forall t \in\{1, \ldots, T\}
\end{aligned}
$$

Equation (3) limits the power reduction of each light individually by PRR, which is defined in order to avoid exorbitance reduction to maintain user comfort.

$$
\begin{aligned}
\sum_{t=1}^{T} P_{(l, t)} & =\operatorname{PRR}_{(l)} \times \sum_{t=1}^{T} \text { init. } P_{(l, t)} \\
& \forall l \in\{1, \ldots, L\}
\end{aligned}
$$

The effective role of $P R R$ will be present more obviously in the results section. According to (3), the total power reduction of each light during all periods cannot exceed a defined value. $P R R$ can make power reduction rigid in several ways, such as a function of time. For instance, it can limit the power reduction of certain lights in only certain periods of time.

In addition to the individual setting of each light, the required illumination for the users can be adjusted based on each room. Equation (4) provides the desired illumination for each room by restricting power reduction.

$$
\begin{gathered}
\sum_{l} P_{(l, r, t)}=\operatorname{Max}_{\text {Red }} R_{(l, r, t)} \times \sum_{l} \text { init. } P_{(l, r, t)} \\
\forall r \in\{1, \ldots, R\} \\
\forall t \in\{1, \ldots, T\}
\end{gathered}
$$

Equation (4) guarantees that users in every room have enough illumination. Although the purpose of the algorithm is the cooperation of all the lights based on their conditions, it should be considered that power reduction in consecutive periods can be annoying for the users because the user may feel illumination reduction for several consecutive periods. Therefore, (5) is assigned to prevent power reduction from certain lights in consecutive periods and all the lights should participate in algorithm goals. It means that the algorithm employs the lights for reducing the desired power reduction in short periods. Then the optimized light in one period can be replaced to other light in different period in order to prevent reducing power of a certain light for several hours.

$$
\begin{gathered}
P_{(l, t)}+P_{(l, t-1)} \leq \operatorname{MaxRed} d_{-} T \\
\forall l \in\{1, \ldots, L\} \\
\forall t \in\{2, \ldots, T\}
\end{gathered}
$$

All of the referred constraints are defined in order to balance the power reduction among all the lights and respect to user convenience.

In the next sections, the impacts of this methodology in real life are demonstrated through a case study and several scenarios.

\section{CASE STUDY}

The illumination system can be considered as the most essential system in any workplace since it directly effects on the efficiency of the users. Hence, the main purpose of this section is to validate the proposed methodology and present its impacts on people's real life.

In this case, there is an office building that includes eight rooms and one corridor containing ordinary office tools such as computers, air conditioners, and lighting system. The lighting system of the building is fluorescent lights that are fully controllable with DALI ballasts. Moreover, several energy meters monitor the energy consumption of the building. In the main core of the SCADA, there are several distributed based Programmable Logic Controllers (PLCs) for managing the consumption. The more detailed information regarding the SCADA model is available on [10].

Fig. 2 illustrates the plan of a part of the building that is controlled by the proposed system.

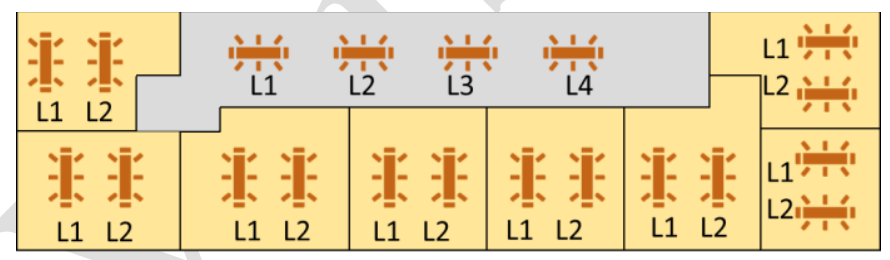

Fig. 2. The plan of the office building considered for the case study.

As can be seen in Fig. 2, in the present office building, each room has two $100 \mathrm{~W}$ lights, and the corridor has four $100 \mathrm{~W}$ lights. Therefore, the present case study considers 20 controllable and reducible lights. If all the lights are turned on in maximum intensity mode, the maximum power consumption of the lighting system in each period is $2000 \mathrm{~W}$. It should be mentioned that the maximum power reduction of each light is bounded to $60 \%$ of its nominal power consumption to avoid turning any light off.

The focus of the algorithm is on 48 periods of the day (12 hours with 15 minutes time slots) from 8:00 AM to 8:00 PM to consider the working hours completely. The utilized data in this section are real data that are monitored from the SCADA system and used in the case study as day-ahead information.

Furthermore, the initial power consumption of the building, initial power consumption of the lights, and the power consumption of the other devices in 48 periods of the case study are shown in Fig. 3-(A). Also, the total power consumption of each light during all periods without any optimization and power reduction is illustrated in Fig. 3-(B).

As can be seen in Fig. 3-(A), the power consumption of the lighting system has been changed during the day and in some periods, such as 1, 2, 5, 6 and 48, the power consumption of the lights is equal to zero. Also, in Fig. 3-(B) the contribution of each light is presented individually. Furthermore, as it is clear in Fig. 3-(B), the total power consumption of lights number 1, 9 , and 10 are lower than the total power consumption of other lights, and therefore, it is expected that they will have lower power reduction too. 

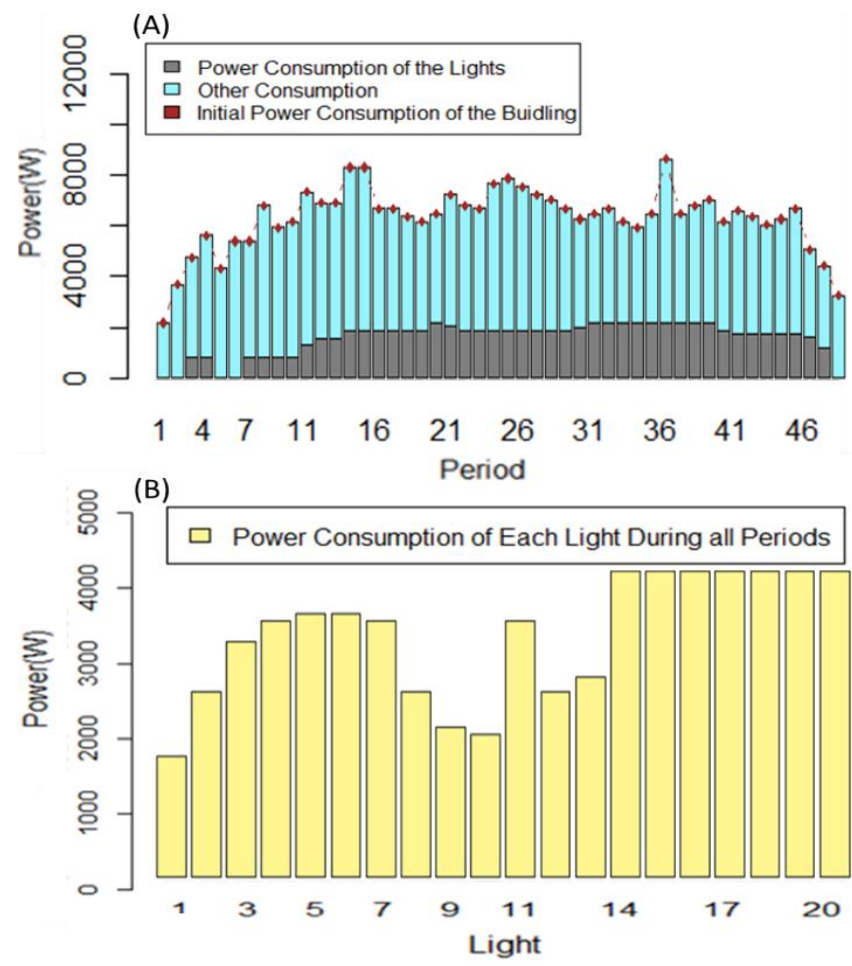

Fig 3. Input data considered for the case study.

Four scenarios are considered for the present case study in order to verify the impact of comfort constraints on the power reduction pattern. It should be noted that in all four scenarios, the initial power consumption of the building and lights, the priority numbers of the lights, and the required power reduction of the system are considered as same as each other.

\section{First Scenario}

In the first scenario, the algorithm is going to be implemented without considering any comfort constraints to obtain the results in a normal situation. It means in the first implementation of the algorithm, (3), (4), and (5) are ignored and the required power reduction should be achieved without considering user satisfaction.

\section{Second Scenario}

In the second scenario, (3) would be involved in the algorithm in order to show the effect of PRR on power reduction. PRR is defined to survey the role of each light in all periods and limit the exorbitance power reduction from each light. PRR can be also assigned for only certain lights in certain periods. The second scenario has two sub-scenarios of A and B that are implemented with general PRR and can be more rigid for certain lights and periods with specific PRR.

\section{Third Scenario}

In the third scenario, a minimum illumination level for each room is considered with involving (4) an algorithm implementation. Regarding the position of each room in the building and taking advantage of natural daylight, the MaxRed_R parameter would be a different percentage of initial power consumption for different rooms.

\section{Fourth Scenario}

The fourth scenario involves (5) in the optimization algorithm implementation to validate the performance with its all comfort constraints. The purpose of (5) is to avoid power reduction from a certain light in consecutive periods, which is important for respecting the user comfort level.

Table I indicates the details of the parameters that are used in the proposed four scenarios.

TABLE I. THE CONSIDERED PARAMETERS FOR DIFFERENT SCENARIOS

\begin{tabular}{|c|c|c|c|c|}
\cline { 2 - 5 } \multicolumn{1}{c|}{} & $\begin{array}{c}\text { PRR } \\
{[\%]}\end{array}$ & $\begin{array}{c}\text { PRR } \\
\text { (Specific) [\%] }\end{array}$ & $\begin{array}{c}\text { MaxRed } \\
\text { R [\%] }\end{array}$ & $\begin{array}{c}\text { MaxRed } \\
\text { T [W] }\end{array}$ \\
\hline 1st Scenario & - & - & - & - \\
\hline 2nd Scenario I & 40 & - & - & - \\
\hline 2nd Scenario II & 40 & 20 & - & - \\
\hline 3rd Scenario & 40 & 20 & 50 & - \\
\hline 4th Scenario & 40 & 20 & 50 & 60 \\
\hline
\end{tabular}

As it can be seen in Table I, in each execution of the algorithm, one comfort constraint is added, and at the end, all the constraints are involved in the algorithm. It should be noted that the considered values in Table I are correlated to present case study, and they could be different values based on the initial situation of the other cases.

As a summary, a case study has been shown in this section in order to validate the performance of the proposed algorithm and the obtained results on different conditions considering various parameters will be shown on the next section.

\section{RESULTS}

This section presents the obtained results of the proposed optimization algorithm in four different scenarios. The main purposes of the presented case study are to reduce a certain amount of power reduction in each period, while the comfort of the users is respected. Therefore, outputs of the algorithm would be the situation of each light in each period as well as the amount of power reduction of each light in each period.

It should be noted that the amount of power reduction in all scenarios is considered equal. Fig. 4 shows the considered power reduction of all lights in each period without any details about participant lights.

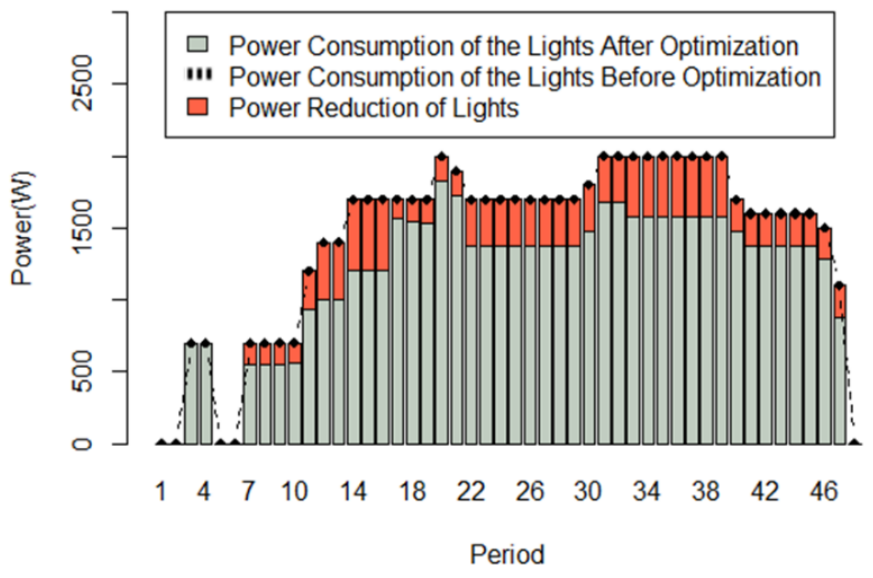

Fig 4. Consumption of the lighting system before and after optimization. 
As Fig. 4 demonstrates, there is various power reduction in each period, somehow the amount of power reduction is constant in all periods while the participated lights would be different considering various conditions. The first result of the algorithm is dedicated to the first scenario while all the comfort constraints are ignored, as Fig. 5 illustrates. The total power reduction of each light during all periods is shown in Fig. 5-(A), and the role of each light in each period is presented in Fig. 5(B). It should be noted that Fig. 5_B illustrates the power reduction of lights related to each room. For instance, L2.5 represents the second light in room number 5 and the corridor has been assumed as $9^{\text {th }}$ room.

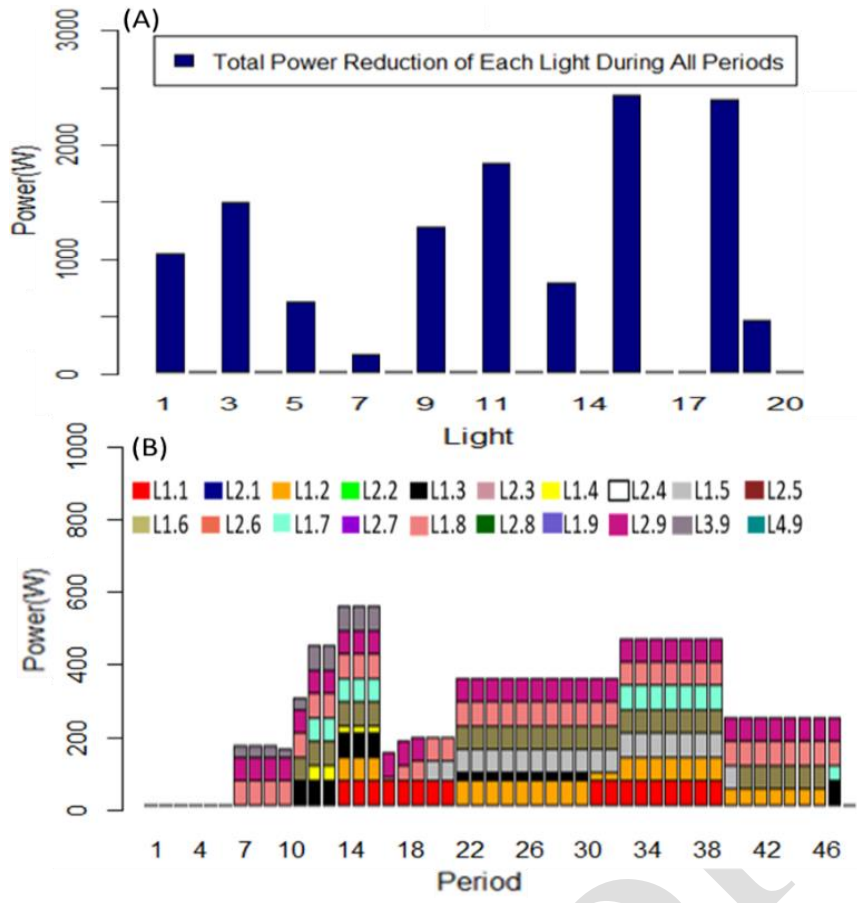

Fig. 5. Optimization results in $1^{\text {st }}$ scenario.

As can be seen in Fig. 5-(A), many lights have not participated in optimization while the other lights such as 15 and 18 have almost $2500 \mathrm{~W}$ power reduction during all periods. Also, in Fig. 5-(B), the required power reduction has been achieved in each period, however, there are some specific lights that are reduced frequently during the case study. This means the user comfort level is definitely distorted in this situation.

Fig. 6 illustrates the situation of power reduction of lights in scenario $2-$ (I) that PRR is considered to avoid exorbitance power reduction. It should be mentioned that PRR is in general mode in this scenario. As it is obvious in Fig. 6-(A), the rate of total power reduction in lights 11,15 , and 18 have been decreased significantly. Comparing to the first scenario, more lights have participated in the optimization process. Also, Fig. 6-(B) verifies that by applying (3) and PRR the power reduction pattern has been changed. However, there are still many successive periods that reduce the power of some specific lights while the others do not participate in the process. The other benefit of defining PRR in the optimization process is to assign the reduction for only certain devices and certain periods. In scenario $2-(\mathrm{II})$, the PRR is applied for some specific lights, and its impact on the output of the optimization is shown in Fig. 7.

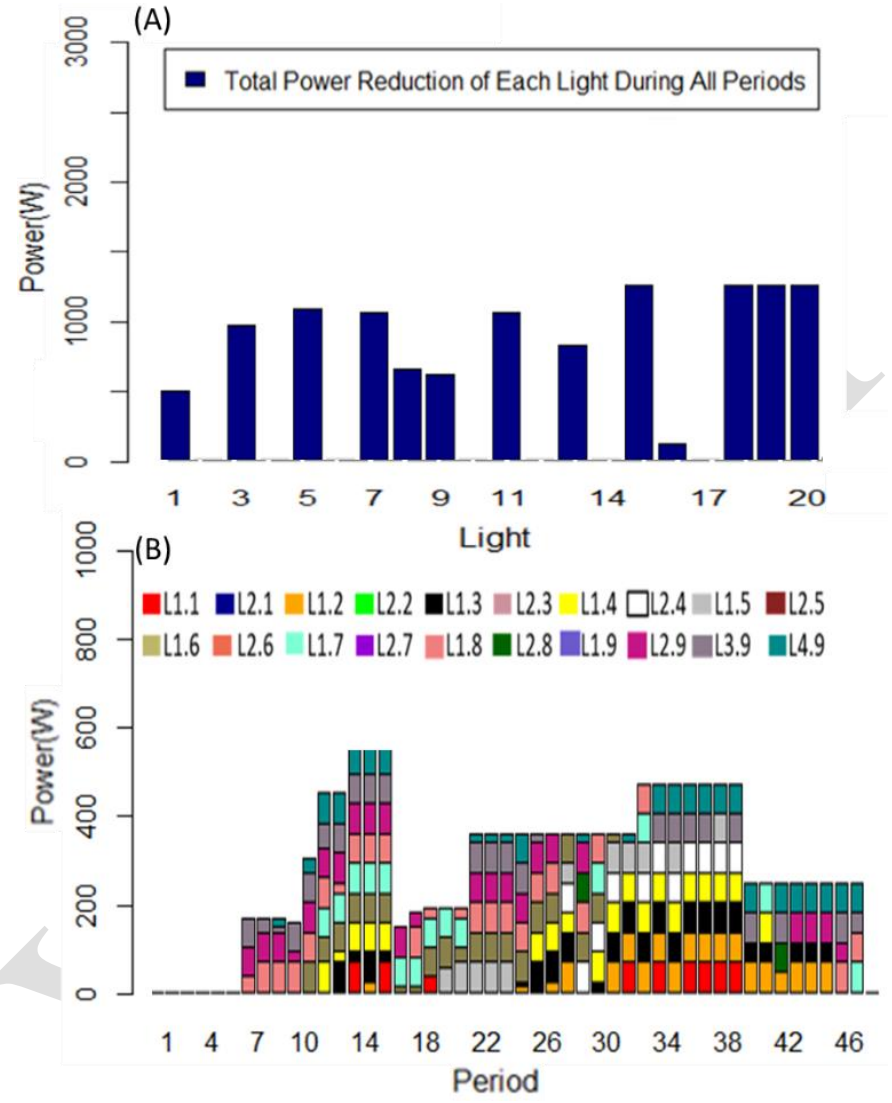

Fig. 6. Optimization outputs for $2^{\text {nd }}$ scenario - (I).

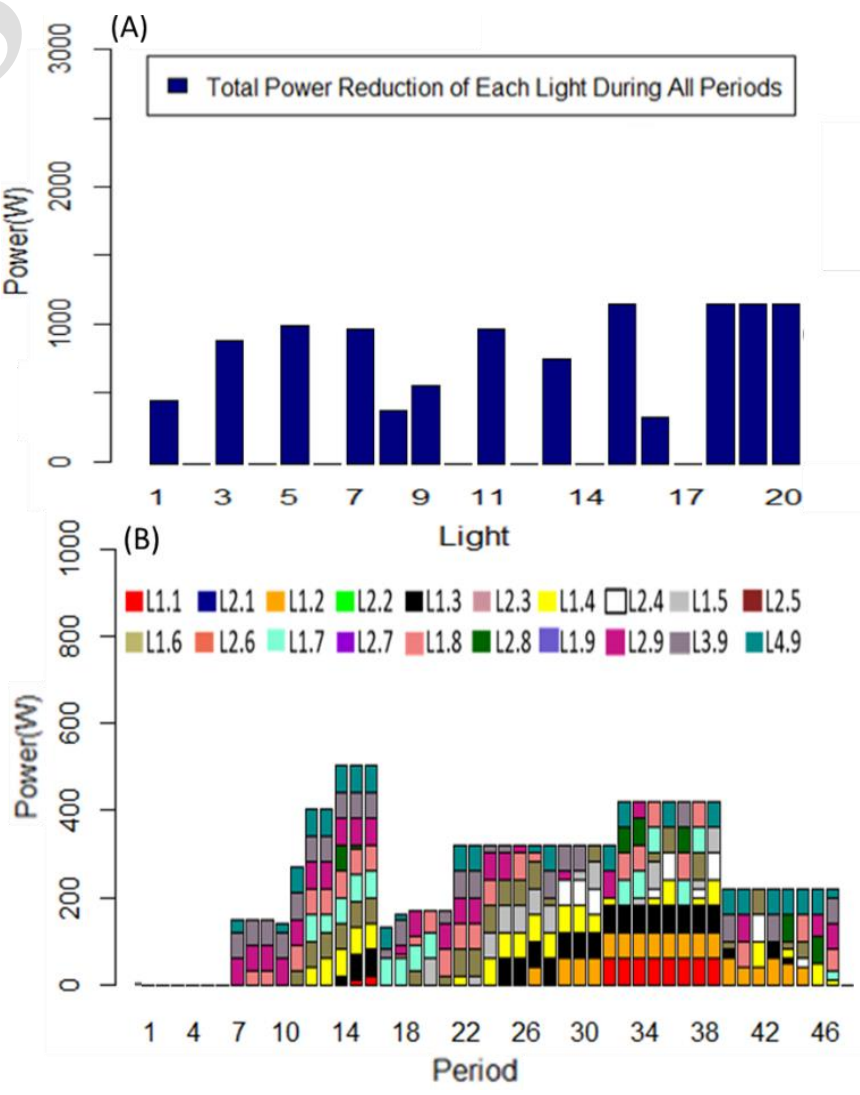

Fig. 7. Optimization outputs for $2^{\text {nd }}$ scenario - (II). 
According to Fig. 7-(A), it can be interpreted that light number 8 is more limited than scenario $2-$ (I) by applying a specific PRR. This limitation can be due to technical reasons or based on defined preferences of the user. The results of third scenario are shown in Fig. 8.
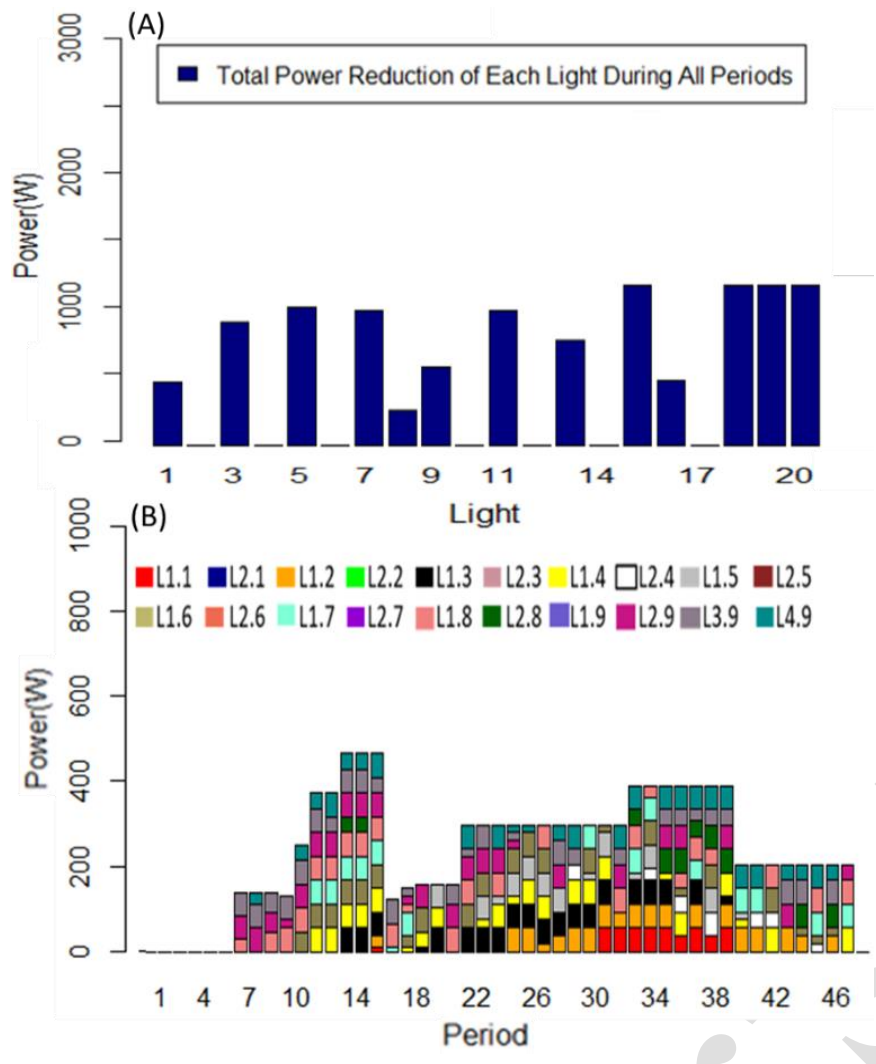

Fig. 8. The obtained results from optimization in $3^{\text {rd }}$ scenario.

As it is clear in Fig. 8-(B), all lights, especially light number 8 and 16, have minor changes comparing with other scenarios. This means in the third scenario, the user comfort level has been more respected, and therefore, the optimization results become more favorable. The last scenario considered all the capabilities validating the effect of (5) on improving this problem. The amount of total power reduction in each light has been reduced, which means the required power reduction of the algorithm is reduced more balanced among the lights. For instance, lights number 4, 6, 14, and 17 proof this matter, somehow, they are did not participate in the previous scenario and they are reduced in this scenario. However, it should be noted that if there are some successive periods to reduce the power of light, the amount of power reduction is insignificant, and it should not be exceeded the amount defined in MaxRed_T.

\section{CONCLUSIONS}

This paper proposed a multi-period optimization algorithm for minimizing the power consumption of the lighting system in an office building. A case study was proposed to validate the performance of the algorithm by applying several levels of user comfort and preferences. The gained results from the case study show that the proposed optimization algorithm is able to reduce the lighting consumption more balanced among the lights considering not reducing from specific lights in successive periods. Therefore, the purpose of the optimization algorithm is reached while the user preferences and comfort level has not been disturbed.

\section{REFERENCES}

H. Ma, J. Lai, C. Li, F. Yang, and Z. Li, "Analysis of school building energy consumption in Tianjin, China," Energy Procedia, vol. 158, pp. 3476-3481, Feb. 2019.

M. Bisadi, A. Akrami, S. Teimourzadeh, F. Aminifar, M. Kargahi, and M. Shahidehpour, "IoT-Enabled Humans in the Loop for Energy Management Systems: Promoting Building Occupants' Participation in Optimizing Energy Consumption," IEEE Electrif. Mag., vol. 6, no. 2, pp. 64-72, Jun. 2018.

M. Tavakoli, F. Shokridehaki, M. Marzband, R. Godina, and E. Pouresmaeil, "A two stage hierarchical control approach for the optimal energy management in commercial building microgrids based on local wind power and PEVs," Sustain. Cities Soc., vol. 41, pp. 332-340, Aug. 2018.

O. Abrishambaf, P. Faria, L. Gomes, J. Spínola, Z. Vale, and J. Corchado, "Implementation of a Real-Time Microgrid Simulation Platform Based on Centralized and Distributed Management," Energies, vol. 10, no. 6, p. 806, Jun. 2017.

"Reschedule of Distributed Energy Resources by an Aggregator for Market Participation," Energies, vol. 11, no. 4, p. 713, Mar. 2018.

D. Xie, L. Yu, T. Jiang, and Y. Zou, "Distributed Energy Optimization for HVAC Systems in University Campus Buildings," IEEE Access, vol. 6, pp. 59141-59151, 2018.

B. Si, Z. Tian, X. Jin, X. Zhou, and X. Shi, "Ineffectiveness of optimization algorithms in building energy optimization and possible causes," Renew. Energy, vol. 134, pp. 1295-1306, Apr. 2019.

F. A. Qureshi, I. Lymperopoulos, A. A. Khatir, and C. N. Jones, "Economic Advantages of Office Buildings Providing Ancillary Services With Intraday Participation," IEEE Trans. Smart Grid, vol. 9, no. 4, pp. 3443-3452, Jul. 2018.

M. Khorram, P. Faria, O. Abrishambaf, and Z. Vale, "Consumption Optimization of an Office Building using Different Approaches," in 2018 IEEE Symposium Series on Computational Intelligence (SSCI), 2018, pp. 1634-1638.

O. Abrishambaf, P. Faria, and Z. Vale, "SCADA Office Building Implementation in the Context of an Aggregator," in 2018 IEEE 16th International Conference on Industrial Informatics (INDIN), 2018, pp. 984-989.

M. Khorram, P. Faria, O. Abrishambaf, and Z. Vale, "Lighting Consumption Optimization in an Office Building for Demand Response Participation," in 2018 Clemson University Power Systems Conference (PSC), 2018, pp. 1-5.

F. Wang et al., "Multi-Objective Optimization Model of SourceLoad-Storage Synergetic Dispatch for a Building Energy Management System Based on TOU Price Demand Response," IEEE Trans. Ind. Appl., vol. 54, no. 2, pp. 1017-1028, Mar. 2018.

M. Khorram, P. Faria, O. Abrishambaf, and Z. Vale, "Demand Response Implementation in an Optimization Based SCADA Model Under Real-Time Pricing Schemes," in International Symposium on Distributed Computing and Artificial Intelligence, 2019, pp. 21-29.

M. Khorram, O. Abrishambaf, P. Faria, and Z. Vale, “Office building participation in demand response programs supported by intelligent lighting management," Energy Informatics, vol. 1, no. 1, p. 9, Dec. 2018.

W. Si, H. Ogai, K. Hirai, H. Takahashi, and M. Ogawa, "An improved PSO method for energy saving system of office lighting," in SICE Annual Conference 2011, 2011.

P. Kar, A. Shareef, A. Kumar, K. T. Harn, B. Kalluri, and S. K. Panda, "ReViCEE: A recommendation based approach for personalized control, visual comfort \&amp; energy efficiency in buildings," Build. Environ., vol. 152, pp. 135-144, Apr. 2019. 